\title{
Mitomycin-C suppresses mucus secretion in an ileal neobladder rat model
}

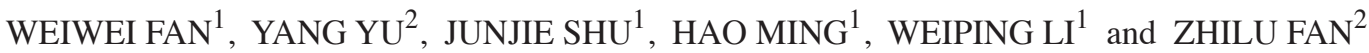 \\ ${ }^{1}$ Dalian Medical University, Dalian, Liaoning 116085; ${ }^{2}$ Department of Urinary Surgery, \\ Second Affiliated Hospital of Dalian Medical University, Dalian, Liaoning 116023, P.R. China
}

Received June 13, 2014; Accepted April 30, 2015

DOI: $10.3892 /$ etm.2015.2529

\begin{abstract}
The aim of the present study was to investigate the mucus secretion status of mature goblet cells following the application of mitomycin-C (MMC) in ileal neobladder rat models. Bladder substitution models were established in Sprague Dawley rats, which had been divided into five groups, namely the control (sham), normal saline(NS), high-dose MMC (HMMC), low-dose MMC (LMMC) and dehydrated alcohol (DA) groups. To evaluate the total protein concentration and level of sialic acid following the therapy, urine from the rats in each group was collected on days 8,11 and 14. In addition, to observe the variances between mucus secretion and the ileum goblet cells, immunohistochemistry and hematoxylin and eosin staining were conducted in the different groups on day 17. The results indicated that the ileal neobladder mucosas in the MMC groups were clearly undamaged, as compared with the DA group. Furthermore, the MMC and DA groups were shown to inhibit the proliferation of goblet cells. The concentration of protein and sialic acid in the LMMC group was found to be lower compared with the NS group, while the concentration in the HMMC group was considerably lower. In conclusion, HMMC was demonstrated to evidently reduce the mucin and sialic acid concentration in the urine, without visible damage to the ileal neobladder mucus membrane. Therefore, MMC may provide a novel therapeutic approach for the treatment of certain bladder conditions.
\end{abstract}

\section{Introduction}

As an effective treatment for invasive bladder tumors, bladder reconstruction, which predominantly comprises ileal neobladder and ileal-colon neobladder in situ, has a serious side effect in that the intestinal mucus secretion function remains (1). Although intestinal villi and microvilli gradually shrink and the number

Correspondence to: Dr Zhilu Fan, Department of Urinary Surgery, Second Affiliated Hospital of Dalian Medical University, 467 Zhongshan Road, Dalian, Liaoning 116023, P.R. China

E-mail: fanzhiludr@yeah.net

Key words: goblet cells, neobladder model, mitomycin-C of goblet cells reduce with time $(2,3)$, complications associated with the orthotopic neobladder in the early postoperative period, caused by a cork of mucus secretion, remain a challenging problem in clinical practice.

Previously, the effects of several antitumor agents on ileum mucus secretion in rats was studied, and the results revealed that mitomycin-C (MMC) inhibited the mucus secretion of goblet cells in vitro (4). In the present study, the administration of different MMC dosages into rat bladder substitution models was investigated with the aim of determining a suitable MMC dosage for local application.

\section{Materials and methods}

Establishment of the rat bladder substitution models. A total of 50 healthy Sprague Dawley (SD) rats (weight, 250 $20 \mathrm{~g}$ ) were purchased from the Experimental Animal Center of Dalian Medical University (Dalian, China). The SD rats were randomly separated into five groups, which included the control or sham operation group (Sham, $n=10$ ), the normal saline (NS) group $(n=10)$, the high-dose MMC (HMMC) group $(0.6 \mathrm{mg} / \mathrm{ml}, \mathrm{n}=10)$, the low-dose MMC (LMMC) group $(0.4 \mathrm{mg} / \mathrm{ml}, \mathrm{n}=10)$ and the dehydrated alcohol (DA) group $(\mathrm{n}=10)$. The animal experiments were approved by the Animal Research Ethics Committee of Dalian Medical University.

The rats received an injection of $5 \%$ glucose and sodium chloride (GNS; Pfizer, Inc., Dalian, China) solution preoperatively at $24 \mathrm{~h}$. In addition, 100,000 units sodium penicillin was injected intramuscularly at $30 \mathrm{~min}$ prior to surgery, after which intraperitoneal anesthesia of $10 \%$ chloral hydrate ( $3 \mathrm{ml} / \mathrm{kg}$ ) was administered. After fixing the rats into position, a catheter was placed into the bladder through the urethra, and the bladder was emptied. The urine was collected and stored at $-80^{\circ} \mathrm{C}$. A middle abdominal incision with a length of $3.5 \mathrm{~cm}$ was made, after which the bladder was found and the cecum was exposed to the appendix and protected with non-sterile (NS) gauzes (Kanjie, Zhejiang, China). A segment of the terminal ileum $(2.5-3.0 \mathrm{~cm})$, that was $2-3 \mathrm{~cm}$ away from the cecum and had an adequate blood supply (with the vascular pedicle), was removed and daubed with iodophor (SigmaAldrich, St. Louis, MO, USA). NS gauzes were again used to protect the surgical site. Ileal continuity was restored and the ileal was put into the abdomen. The two ends of the pedicled ileal segment were closed and an arc incision measuring $\sim 1 \mathrm{~cm}$ 
in length was made at the central part of the bowel loops in the mesenteric margin (vascular pedicle contra lateral). The majority of the bladder was removed up until $0.3 \mathrm{~cm}$ away from the bladder triangle, and the arc margin of the neobladder was connected with the remaining bladder (needle distance, $1 \mathrm{~mm}$ ). The abdominal cavity was closed and the surgery time was recorded (Fig. 1). After $6 \mathrm{~h}$ of fasting, the rats were fed $5 \%$ GNS solution by injection, and subsequently received a normal diet at $24 \mathrm{~h}$ after the surgery. In addition, the rats were administered anti-infective therapy (cefminox, $20 \mathrm{mg} / \mathrm{kg} \cdot$ day) for 3 days. With regard to the sham-operated group, the incision was closed following the opening of the cavity, with the remaining procedure the same as aforementioned.

Collection of urine and tissue specimens. Urine specimens were collected prior to the irrigation of the ileal neobladder on days 8, 11, 14 and 17. Intraperitoneal anesthesia was induced using $10 \%$ chloral hydrate $(3 \mathrm{ml} / \mathrm{kg})$, and the rats were fixed in position. Urine was collected by inserting a catheter into the bladder through the urethra. The upper fluid layer was separated by centrifugation for $5 \mathrm{~min}$ at room temperature, and the specimens were stored at $-80^{\circ} \mathrm{C}$. On day 17 , following urine specimen collection, a mid-abdominal incision was made to retrieve the substituted bladder and normal ileum tissues. The specimens were divided into two pieces, one section was stored at $-80^{\circ} \mathrm{C}$ and the other section was fixed in $4 \%$ formalin.

Bicinchoninic acid (BCA) assay of the urine protein concentration. A BCA protein assay kit was purchased from Pierce Biotechnology, Inc. (Rockford, IL, USA). Urine samples containing 0.2-50 $\mu \mathrm{g} / \mu \mathrm{l}$ protein were established, and $1 \mathrm{ml}$ standard working solution from the BCA kit was added to each $20-\mu 1$ sample and mixed. The solutions were incubated for $30 \mathrm{~min}$ at $60^{\circ} \mathrm{C}$, after which the samples were cooled for a minimum of $1 \mathrm{~h}$ and the absorbance was measured at $562 \mathrm{~nm}$ using a visible light spectrophotometer (Thermo Fisher Scientific, Waltham, MA, USA). A standard curve of absorbance against protein $(\mu \mathrm{g})$ was constructed, and the amount was determined from the curve. Subsequently, the protein concentration in the urine was determined.

Assay of sialic acid in the urine. A sialic acid assay kit (Abcam, Cambridge, UK) was used to measure the concentration of sialic acid in the urine. Briefly, $0.1 \mathrm{ml}$ periodic acid $(0.04 \mathrm{M})$ was added to the samples and thoroughly stirred, after which the samples were incubated in an ice bath for $20 \mathrm{~min}$. Next, $1.25 \mathrm{ml}$ resorcinol reagent was added and the samples were placed in an ice bath for a further $5 \mathrm{~min}$. The solution was heated at $100^{\circ} \mathrm{C}$ for $15 \mathrm{~min}$ and cooled in tap water, following which $1.25 \mathrm{ml}$ tertbutyl alcohol was added and mixed vigorously to produce a single-phase solution. The sample tubes were placed in a $37^{\circ} \mathrm{C}$ water bath for $3 \mathrm{~min}$ to stabilize the color, and the absorbance was read at $560 \mathrm{~nm}$.

Hematoxylin and eosin $(H \& E)$ and Alcian blue-periodic acid Schiff (AB-PAS) staining. Neobladder specimens were formalin-fixed and embedded in paraffin, and $H \& E$ staining was performed as previously described $(3,4)$. A scoring system was used to categorize the mucus membrane of the intestines, which was evaluated independently by two pathologists who were blinded to the specimen data. The scoring system was recorded as follows: 0 , normal tissue; 1 , slight intestinal epithelium desquamation that is restricted to the mucus membrane cupula; 2, villi necrosis that is restricted to the mucus membrane cupula; 3 , central villi; 4 , complete villi necrosis.

With regard to the AB-PAS staining, paraffin-embedded sections were treated with Schiff reagents, as previously described. Sialic acid mucus protein appeared as a blue stain (4). Five villi in every sample were selected and the number of goblet cells with sialic acid mucus were counted using a microscope (Olympus Corporation, Tokyo, Japan), along with the total number of goblet cells, to acquire the mean value.

Immunohistochemical staining. Immunohistochemical staining of the specimens was performed using an anti-rat mucin 2 (MUC-2) antibody (AB11197; Santa Cruz Biotechnology, Inc., Santa Cruz, CA, USA), as previously described (4). The expression of MUC-2 was observed as buffy particles in the cytoplasm of the goblet cells.

Statistical analysis. Data were analyzed using SPSS software, version 19.0 (IBM SPSS, Armonk, NY, USA), and are presented as the mean \pm standard deviation. Statistical significance was determined by one-way analysis of variance, the Student's t-test, the Kruskal-Wallis test and the $\chi^{2}$ test, where $\mathrm{P} \leq 0.05$ was considered to indicate a statistically significant difference.

\section{Results}

Morphological effects of the ileal neobladder and normal ileal mucus membrane in the different groups. Damage to the villi on the small intestinal structures was most significant in the DA group. The mucus and submucus membrane understructure exhibited varying degrees of destruction, while the muscular layer exhibited tears and hemorrhage. When compared with the DA group, the damage caused by HMMC and LMMC was minimal. The majority of the mucus and submucus membrane and the muscular layer remained normal. However, in the LMMC, HMMC and DA groups, the goblet cells were predominantly scattered in the root of villus, and did not present with the typical goblet shape (Fig. 2)

Scoring of the ileal neobladder and normal ileal mucus membrane revealed that the average scores of the HMMC and LMMC groups were much lower compared with those of the DA group $(\mathrm{P}<0.01)$, which indicated that $\mathrm{MMC}$ resulted in significantly less damage compared with DA.

Effects on goblet cells in the different groups. AB-PAS staining results revealed that the quantity of goblet cells in the HMMC and LMMC groups increased when compared with the sham and NS groups $(\mathrm{P}<0.05)$; however the percentage of mature goblet cells decreased $(\mathrm{P}<0.05$; Table I).

Urine protein concentrations in the different groups. Urine protein concentrations in the HMMC and DA groups on days 11, 14 and 17 following surgery were shown to decrease when compared with the NS group $(\mathrm{P}<0.05)$. In the LMMC 
A

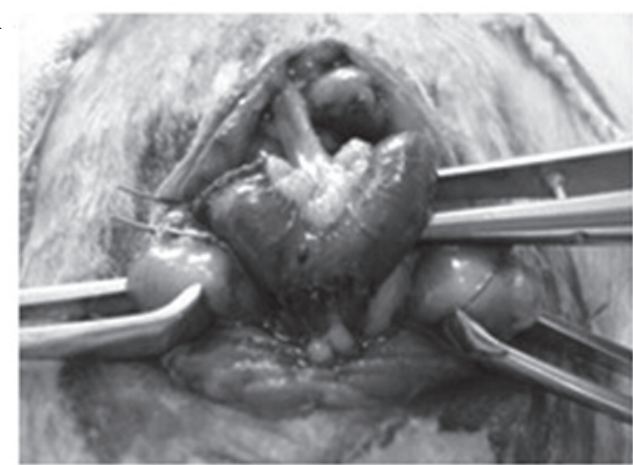

C

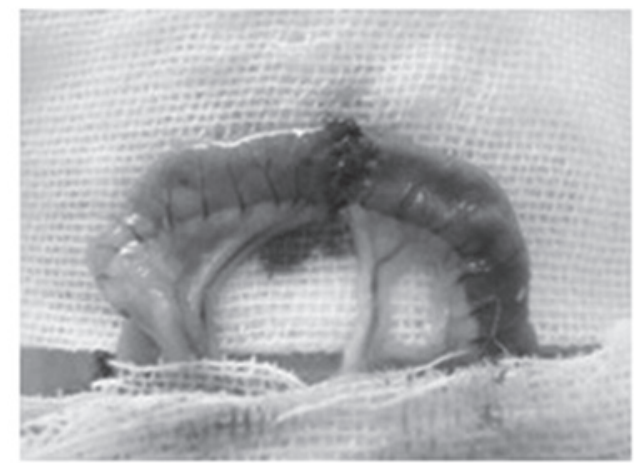

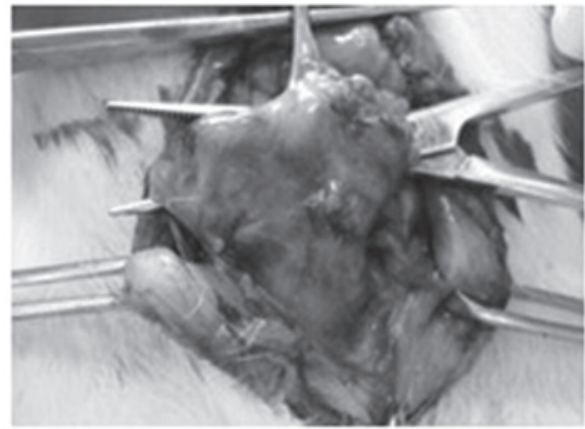

D

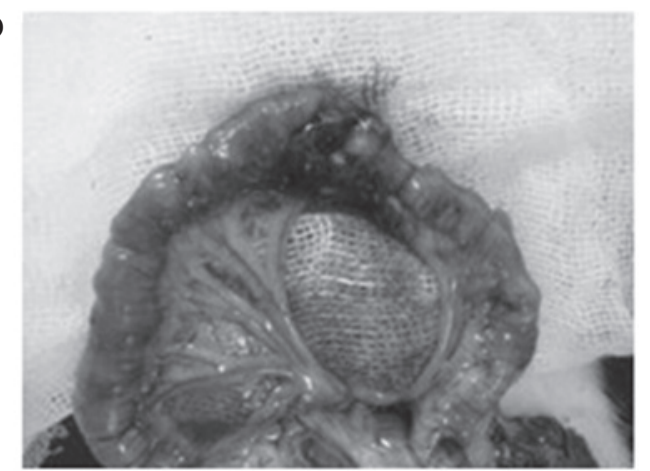

Figure 1. Rat bladder substitution models. (A) U-shaped anastomose between the ileal loops and the rest of the bladder. (B) Two anastomosed ileal ends. (C) Two weeks subsequent to the bladder substitution. (D) Two weeks subsequent to the ileal ends being anastomosed.

A

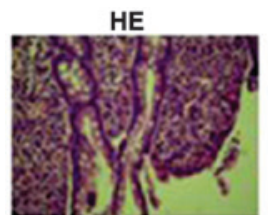

C

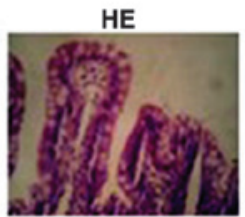

AB-PAS

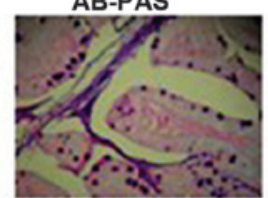

AB-PAS

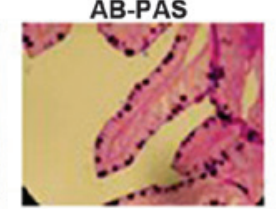

$\mathbf{E}$

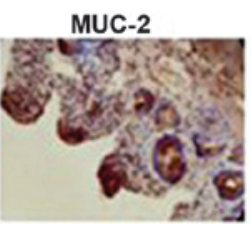

MUC-2

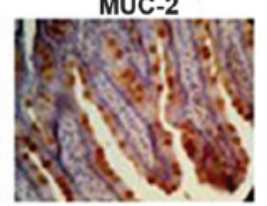

B

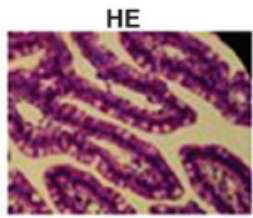

D

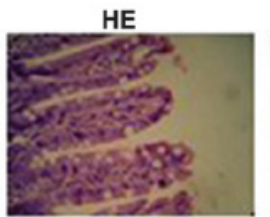

AB-PAS

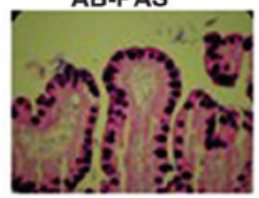

AB-PAS

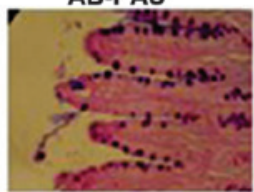

MUC-2

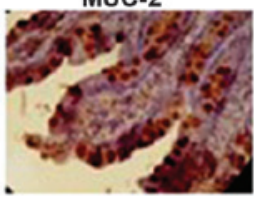

MUC-2

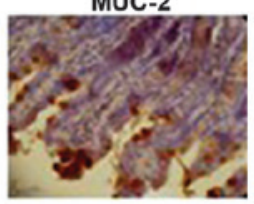

MUC-2

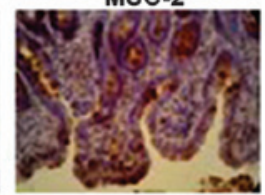

Figure 2. Morphological effects of the ileal neobladder and normal ileal mucus membrane in the (A) dehydrated alcohol, (B) high-dose mitomycin-C, (C) low-dose mitomycin-C, (D) normal saline and (E) sham groups. HE staining revealed the goblet cells with white vacuoles. AB-PAS staining revealed the goblet cells as a blue stain. MUC-2 immunohistochemistry stained the goblet cells with brown coloration. HE, hematoxylin and eosin; AB-PAS, Alcian blue-periodic acid Schiff; MUC-2, mucin-2.

group, the urine protein concentrations decreased on days 14 and 17 following surgery when compared with the NS group $(\mathrm{P}<0.05)$. When compared with the LMMC group, the urine protein concentrations in the HMMC group on days 11 and 17 following surgery were significantly lower $(\mathrm{P}<0.05$; Table II).

Urine sialic acid concentrations in the different groups. When compared with the NS group, the sialic acid concentration in the HMMC, LMMC and DA groups were significantly different on days 11,14 and 17 following surgery, respectively $(\mathrm{P}<0.05)$. Compared with the LMMC group, the urine sialic acid concentration in the HMMC group on day 17 following surgery was significantly lower $(\mathrm{P}<0.05)$.

\section{Discussion}

To date, numerous methods have been adopted to prevent early mucus plug formation following orthotopic neobladder urinary 
Table I. Quantities of goblet cells in the ileal neobladder mucus villi.

\begin{tabular}{|c|c|c|c|c|}
\hline \multirow[b]{2}{*}{ Group } & \multirow[b]{2}{*}{ Cases (n) } & \multicolumn{3}{|c|}{ AB-PAS coloring } \\
\hline & & $\begin{array}{l}\text { Blue-colored } \\
\text { cells }(n)\end{array}$ & $\begin{array}{c}\text { Total } \\
\text { number (n) }\end{array}$ & $\begin{array}{c}\text { Blue-colored/total } \\
\text { number }(\%)\end{array}$ \\
\hline NS & 10 & $13.63 \pm 2.33$ & $32.63 \pm 6.26^{\mathrm{a}}$ & $42.96 \pm 9.51^{\mathrm{a}}$ \\
\hline HMMC & 10 & $5.88 \pm 1.25$ & $38.10 \pm 5.89^{b}$ & $15.73 \pm 4.10^{\mathrm{b}}$ \\
\hline LMMC & 10 & $7.75 \pm 8.82$ & $38.63 \pm 5.95^{\mathrm{b}}$ & $20.90 \pm 5.74^{\mathrm{b}}$ \\
\hline DA & 10 & $3.13 \pm 1.13$ & $1.14 \pm 0.35^{\mathrm{a}, \mathrm{b}}$ & $38.33 \pm 10.80^{\mathrm{a}}$ \\
\hline
\end{tabular}

${ }^{\mathrm{a}} \mathrm{P}<0.05$, vs. HMMC group. ${ }^{\mathrm{b}} \mathrm{P}<0.05$, vs. sham group. Data are presented as the mean \pm standard deviation. AB-PAS, Alcian blue-periodic acid Schiff; NS, normal saline; HMMC, high-dose mitomycin-C; LMMC, low-dose mitomycin-C; DA, dehydrated alcohol.

Table II. Changes in the urine protein concentrations following surgery ( $\mathrm{mg} / \mathrm{ml})$.

\begin{tabular}{lcccccc}
\hline Group & Cases (n) & Preoperative & Day 8 & Day 11 & Day 14 & Day 17 \\
\hline Sham & 10 & $0.035 \pm 0.0063$ & $0.046 \pm 0.0069$ & $0.044 \pm 0.0060$ & $0.040 \pm 0.0059$ & $0.041 \pm 0.0062$ \\
NS & 10 & $0.043 \pm 0.0068$ & $7.91 \pm 1.30$ & $9.19 \pm 1.35^{\mathrm{a}}$ & $10.31 \pm 1.85^{\mathrm{a}, \mathrm{b}}$ & $11.63 \pm 2.31^{\mathrm{a}, \mathrm{b}}$ \\
HMMC & 10 & $0.041 \pm 0.060$ & $8.27 \pm 1.63$ & $6.18 \pm 1.14^{\mathrm{b}, \mathrm{c}}$ & $5.20 \pm 1.33^{\mathrm{b}, \mathrm{c}}$ & $4.16 \pm 0.97^{\mathrm{b}, \mathrm{c}}$ \\
LMMC & 10 & $0.045 \pm 0.0080$ & $8.41 \pm 1.04$ & $7.38 \pm 0.84^{\mathrm{a}, \mathrm{b}}$ & $6.11 \pm 1.04^{\mathrm{b}, \mathrm{c}}$ & $5.59 \pm 0.73^{\mathrm{a}, \mathrm{b}, \mathrm{c}}$ \\
DA & 10 & $0.040 \pm 0.0098$ & $8.53 \pm 1.94^{\mathrm{c}}$ & $5.96 \pm 1.04^{\mathrm{b}-\mathrm{c}}$ & $3.82 \pm 1.61^{\mathrm{a}, \mathrm{b}, \mathrm{c}}$ & $2.56 \pm 1.08^{\mathrm{a}, \mathrm{b}, \mathrm{c}}$ \\
\hline
\end{tabular}

Data are presented as the mean \pm standard deviation. ${ }^{\mathrm{a}} \mathrm{P}<0.05$, vs. $\mathrm{HMMC}$ group; ${ }^{\mathrm{b}} \mathrm{P}<0.05$, vs. postoperative day 8 ; ${ }^{\mathrm{c}} \mathrm{P}<0.05$, vs. sham group. NS, normal saline; HMMC, high-dose mitomycin-C; LMMC, low-dose mitomycin-C; DA, dehydrated alcohol.

diversion surgery. In one method, pure saline is repeatedly injected into a new bladder through a large-diameter catheter or fistula to prevent the accumulation of mucus. In addition, the dissolution of mucus can be promoted through the intravesical instillation of urea to disrupt the mucin molecule hydrogen bonds and to reduce the viscosity of mucin (5). Furthermore, a variety of agents, such as ethanol, formaldehyde, silver nitrate and octreotide, have been reported to reduce mucus secretion (6-8). Early mucus plug formation may also be prevented through the mechanical removal of intestinal mucosa (9), or through the use of an artificial bladder (10).

The intestinal epithelium of mammals is composed of five types of cell (3), including absorption, goblet, Paneth, undifferentiated and endocrine cells. The proliferation and mitosis of goblet cells are active, and the cytotoxic function of MMC is much greater for cells with short cellular cycles. Therefore, the present study was designed on the basis that MMC is more suitable for goblet cells, and the toxic effect for other epithelial cells is weaker. Thus, investigations into the effects on goblet cells can be performed without damaging other cell types.

The H\&E staining results revealed that DA inflicts serious damage to the intestinal epithelium within a short time period, indicating that DA is not an ideal bladder instillation agent. By contrast, the intestinal mucosa injury of the HMMC and LMMC groups was confined to the upper intestinal villi, while the glands and muscles remained normal. The scores from the ileum mucosa Kruskal-Wallis tests have further confirmed these conclusions.
The results of the present study demonstrate that urine protein levels in the HMMC and LMMC groups tend to decrease following the addition of MMC into the bladder, and the degree is associated with the dose. By contrast, the protein concentration in the NS group increased. Urine protein concentrations in the normal bladder tissues were lower compared with those in the experimental groups; thus, it was concluded that the protein concentration in the urine can reflect the level of mucus secretion; the lower the concentration, the lower the level of secretion.

Sialic acid has an evident effect on the space conformation of protein molecules (11), and the amount of sialic acid reflects the maturity of goblet cells $(12,13)$. Previous studies have indicated that the use of enzymes or a combination of acid hydrolysis can significantly reduce the viscosity of mucus $(14,15)$. The results of the present study demonstrated that experimental dosages of 0.6 and $0.4 \mathrm{mg} / \mathrm{ml} \mathrm{MMC} \mathrm{can}$ decrease the level of sialic acid in the urine and the number of mature goblet cells, with the effect dose-dependent. Decreasing the sialic acid level results in decreased mucosity of the mucus, which subsequently prevents early mucus plug formation following surgery.

$\mathrm{H} \& \mathrm{E}$ and AB-PAS histochemical staining revealed that the HMMC and LMMC groups exhibited intestinal epithelium swelling, hyperplasia and hypertrophy of the goblet cells when compared with the NS group. According to these results, the mechanisms underlying the phenomenon were summarized as follows. Firstly, MMC may interfere with the cell cycle of goblet cells, causing mitosis to be blocked at the late $G_{1}$ and early $\mathrm{S}$ phase, and the cell volume to increase. In addition, the 
quantity of immature goblet cells was elevated, which further indicated that MMC can inhibit the development of goblet cells. Secondly, MMC may reduce mucin exocytosis by disturbing organelles. In the urine environment, under the premise of surgical stress, MMC inhibits the DNA replication of goblet cells by provoking the cross-linking of DNA, and the inhibition of DNA replication can lead to a dysfunction of the endoplasmic reticulum, Golgi apparatus and other organelles. Subsequently, protein molecules are no longer assembled properly and are not transferred out of the cells. The aforementioned changes may also be the result of ischemia and anoxia; thus, enlargement and swelling of the goblet cells are observed.

In conclusion, the establishment of experimental models in the present study was simple and time effective. When compared with the DA group, the MMC dosages administered were shown to decrease the early postoperative mucus secretions, with the effect associated with the dose and the percentage of mature goblet cells. However, the mechanism underlying the increased size and immaturity of the goblet cells in the ileal neobladder remains unclear and requires further investigation.

\section{References}

1. Kashif KM and Holmes SA: The use of small intestine in bladder reconstruction. Int Urogynecol J Pelvic Floor Dysfunct 9: 275-280, 1998

2. Sun X, Song M, Bai R, Cheng S, Xing Y, Yuan H, Wang P and Zhou L: Ileal interposition surgery-induced improvement of hyperglycemia and insulin resistance in Goto-Kakizaki rats by upregulation of TCF7L2 expression. Exp Ther Med 5: 1511-1515, 2013.
3. Hautmann RE, de Petriconi RC and Volkmer BG: Lessons learned from 1,000 neobladders: The 90-day complication rate. J Urol 184: 990-994, 2010.

4. Yu Yang: The experimental studies of anti-tumor agents to intestinal mucus secretion in vitro (unpublished $\mathrm{PhD}$ thesis). Dalian Medical University, 2006 (In Chinese).

5. Demirbilek S, Uğuralp S, Gürbüz N, Sezgin N and Kirimlioĝlu H: The use of silver nitrate for chemical de-epithelialization and urothelialization of intestine in a rabbit model of augmentation cystoplasty. Urol Res 31: 236-241, 2003.

6. Leknes IL: Histochemical study on the intestine goblet cells in cichlid and poecilid species (Teleostei). Tissue Cell 42: 61-64, 2010.

7. Khorrami MH, Salehi P, Nouri-Mahdavi K, Ghalamkari A and Tadayyon F: Dramatic effect of a somatostatin analogue in decreasing mucus production by the intestinal segment after enterocystoplasty. J Urol 180: 2501-2503, 2008.

8. Zhang T and Zhou X: Clinical application of expectorant therapy in chronic inflammatory airway diseases (Review). Exp Ther Med 7: 763-767, 2014.

9. Falke G, Caffaratti J and Atala A: Tissue engineering of the bladder. World J Urol 18: 36-43, 2000.

10. Loeffler M, Birke A, Winton D and Potten C: Somatic mutation, monoclonality and stochastic models of stem cell organization in the intestinal crypt. J Theor Biol 160: 471-491, 1993.

11. Shen G-J, Datta AK, Izumi M, Koeller KM and Wong C-H: Expression of $\alpha 2,8 / 2,9$-polysialyltransferase from Escherichia coli K92. Characterization of the enzyme and its reaction products. J Boil Chen 274: 35139-35146, 1999.

12. Levine MJ, Aguirre A, Hatton MN and Tabak LA: Artifical salivas: Present and future. J Dent Res 66: 693-698, 1987.

13. Matsuo K, Ota H, Akamatsu T, Sugiyama A and Katsuyama T: Histochemistry of the surface mucous gel layer of the human colon. Gut 40: 782-789, 1997.

14. Baylin SB: Reversal of gene silencing as a therapeutic target for cancer-roles for DNA methylation and its interdigitation with chromatin. Novartis Found Symp 259: 226-233, 2004.

15. Ozdemir F, Altinisik J, Karateke A, Coksuer H and Buyru N: Methylation of tumor suppressor genes in ovarian cancer. Exp Ther Med 4: 1092-1096, 2012. 